\title{
Odontalgia atípica crónica por leve fisura de la corona de un diente, no apreciable con radiografía
}

\author{
González-González JM*
}

\section{RESUMEN}

Las odontalgias atípicas crónicas obligan en muchos casos al paciente a ir saltando por consultas de diferentes especialistas, sin encontrar la causa, ni el tratamiento apropiado a su padecimiento.

Presentamos tres casos clínicos en los cuales queda demostrada como causa de una odontalgia atípica crónica una fisura de la corona del diente. Pensamos que dicha fisura, cuando se prolonga en profundidad, puede afectar a dentina o pulpa y ser el motivo de ese dolor atípico durante incluso años, terminando en más de un caso en fractura del diente.

Proponemos un diagnóstico temprano de este tipo de etiología, realización de una endodoncia y colocación de una funda metal-porcelana de protección.

Palabras clave: Odontalgia atípica crónica. Dolor facial atípico. Neuralgia trigeminal. Dolor dental. Fisura. Fractura. Diagnóstico. Tratamiento.

\begin{abstract}
In many cases chronic atypical odontalgia make patients visit several different specialists' surgeries, being unable to find the reason or the suitable treatment for the pain.

We present three clinical cases in which a fissure in the tooth crown is proved to be the cause of the chronic atypical odontalgia. We think that when this fissure deepens it can affect dentin or pulp and thus not only cause this atypical pain for years but also result in tooth fracture in more than one case.

We propose an early diagnosis of this type of etiology, the performance of an endodontic treatment and the placement of a protective metal-porcelain cap.
\end{abstract}

Key words: Chronic atypical odontalgia. Atypical facial pain. Trigeminal Neuralgia. Dental pain. Fissure. Fracture. Diagnosis. Treatment.

* Doctor en Medicina y Cirugía. Médico estomatólogo. Práctica privada en Salamanca.

González-González JM. Odontalgia atípica crónica por leve fisura de la corona de un diente, no apreciable con radiografía. Av. Odontoestomatol 2003; 19-6: 271-274

\section{INTRODUCCIÓN}

Existen neuralgias trigeminales, denominadas "idiopáticas" en las que no se ha encontrado una causa aparence y que algunos autores han demostrado finalmente ser causadas por osteomielitis maxilares crónicas (1). Existen otros tipos de dolores poco localizados, continuos, de intensidad variable, sin distribución anatómica clara ni limitación al territorio de inervación de un nervio, en los cuales tampoco fue hallada causa alguna y que han sido clasificados como "dolor facial atípico". En este tipo de dolor hay 
autores que dan especial importancia al factor psicológico, enfocando el tratamiento en este sentido (2), pero esta opinión no siempre es compartida por la generalidad de profesionales (3). Lo cierto es que el paciente va recorriendo numerosas consultas de diferentes especialistas sin encontrar la causa de su dolor en ninguna de ellas (4). En algún caso también se ha sugerido como causa de la odontalgia atípica un dolor neuropático de la cavidad oral, con un componente de dolor mantenido simáticamente (5).

Por otro lado, ha sido descrita una diferente morfología de surcos y fisuras en la superficie dentaria. Algunos de estos surcos eran poco perceptibles en la inspección visual, pero observados a microscopía electrónica de barrido resultaron ser muy profundos, sugiriéndose en tales casos un mayor riesgo de caries (6). La profundidad y posible lesión en el fondo de este tipo de surcos y fisuras ha resultado según diferentes autores, imposible de determinar desde la posición habitual de trabajo con radiografías de aleta, radiovisiografía, lupas, tinciones o con el uso de una sonda exploradora, pues son en muchos casos excesivamente estrechas y sólo apreciables a microscopía (7).

Pensamos que buena parte de las odontalgias atípicas en las que no se halla una causa aparente pueden tener su origen en esas profundas y estrechas fisuras naturales de algunos dientes, que cuando se prolongan llegan a afectar a tejido dentario sensible, dentina o pulpa, duelen entonces durante un tiempo largo incluso de años, y que son el paso previo a una posterior fractura del diente. Presentamos tres casos diagnosticados en un principio como odontalgias atípicas crónicas que eran en realidad producidas por una aparente leve fisura de la corona del diente y exponemos su tratamiento.

\section{CASOS CLÍNICOS}

\section{Caso 1}

Mujer de 56 años de edad con dolor leve e inconstante en hemiarcada superir derecha hace trece meses, pero que se ha agudizado en la última semana, haciendo pensar incorrectamente a la paciente que su origen estaba en el $1^{\circ}$ molar superior derecho. En la inspección sólo se aprecia fisura mesio-distal de la corona del $1^{\circ}$ premolar superior derecho (Fig. 1).

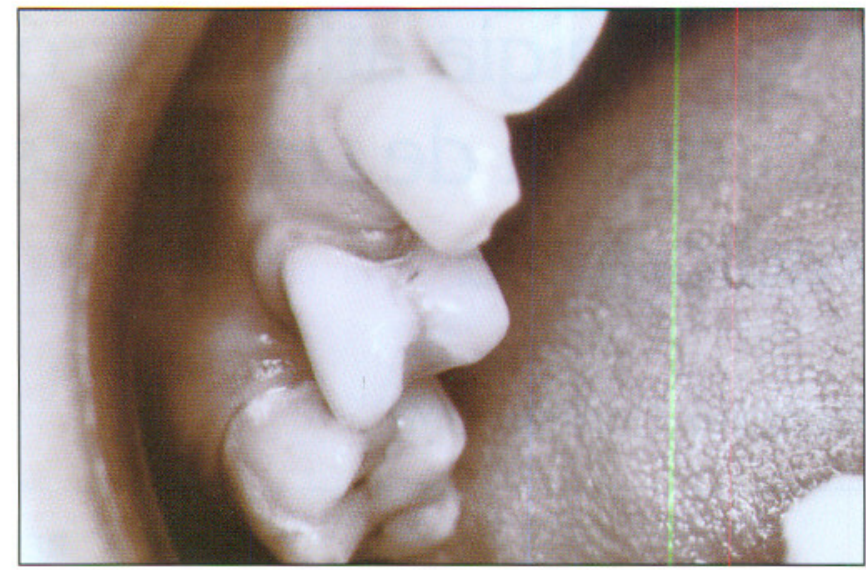

Fig. 1. Fisura medio-distal en la corona del $1^{\circ}$ premolar superior derecho.

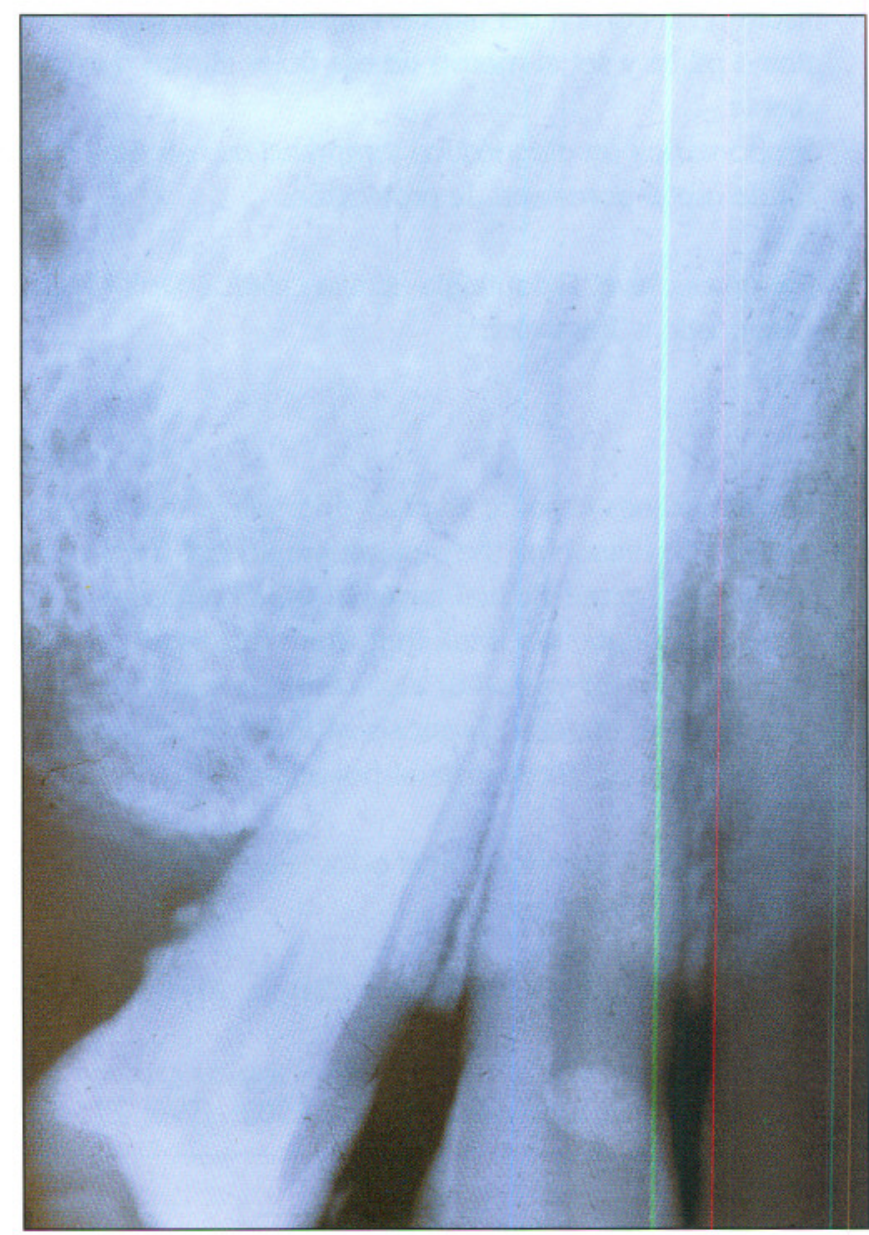

Fig. 2. Imagen radiográfica del $1^{\circ}$ premolar superior derecho en la que no se aprecia signo alguno de fisura coronal.

En la exploración radiográfica (Fig. 2) no hallamos nada. El tratamiento de urgencia fue: apertura cameral de dicho premolar y colocación de un medi- 


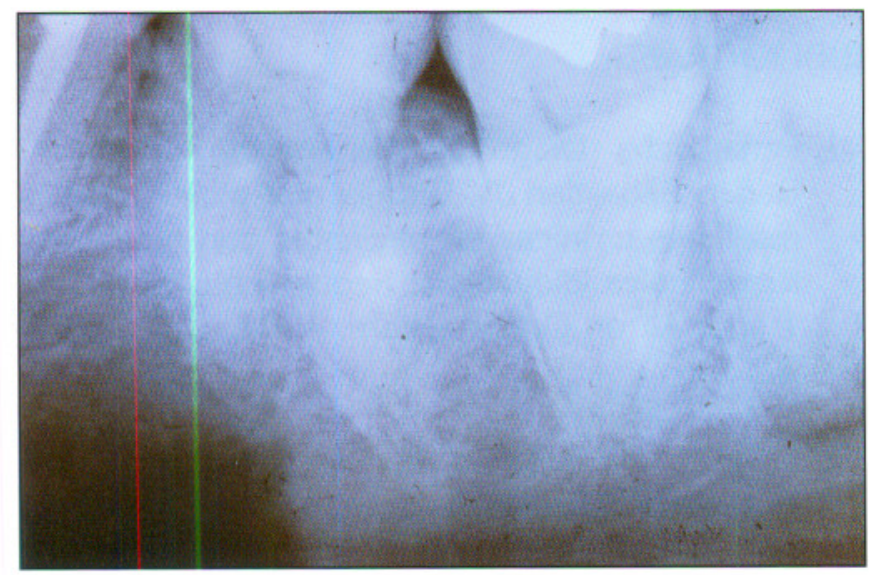

Fig. 3. Imagen radiográfica del $2^{\circ}$ molar inferior izquierdo, en la que no hallamos esa fisura mesio-distal de la corona, que sí habíamos visto previamente durante la exploración.

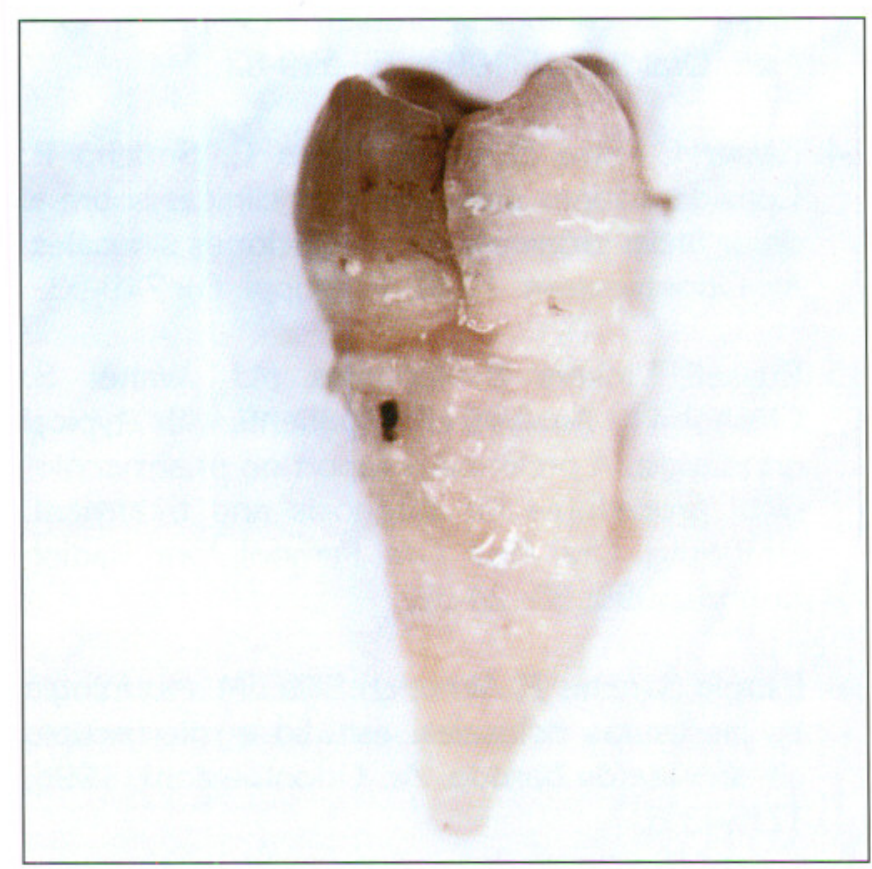

Fig. 4. Segundo molar inferior izquierdo posterior a la extracción. Obsérvese la fractura mesio-distal de su corona.

camente en su interior (Cresophene, Septodont) tapado provisionalmente con un cemento (Cavit, Espe). La odontalgia desapareció y posteriormente se planificó hacer una endodoncia y colocación de una corona metal-porcelana de protección.

\section{Caso 2}

Mujer de 53 años de edad que se presenta en consulta por dolor ocasional en hemiarcada inferior izquierda. Manifestó tener que tomar diversos analgésicos y/o antiinflamatorios en momentos de agu-

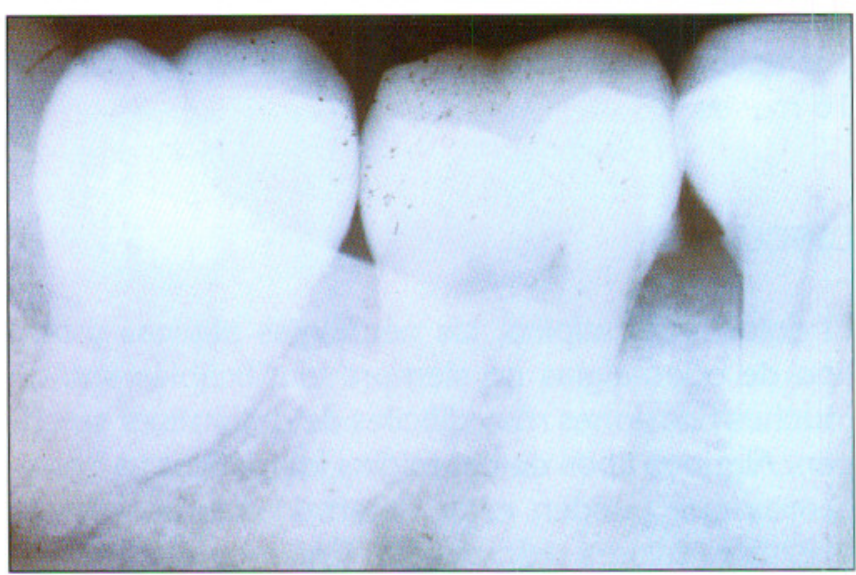

Fig. 5. Imagen radiográfica del segundo molar inferior derecho, en la que no ser observa ningún tipo de fisura coronal.

dización. En la inspección apreciamos un fisura mesio-distal en la corona del $2^{\circ}$ molar inferior izquierdo. En la exploración radiográfica no hallamos nada especial (Fig. 3). Solucionamos el dolor del momento con apertura cameral de dicho diente, colocación de un medicamento (Cresophene, Septodont) y cierre con un cemento provisional (Cavit, Espe). Propusimos hacer posteriormente una endodoncia y colocación de una corona metal-porcelana. El día que habíamos planificado hacer la endodoncia vino con el diente ya fracturado e hicimos la extracción. El diente observado en sentido distomesial permite apreciar dicha fractura (Fig. 4).

\section{Caso 3}

Mujer de 54 años de edad, que presenta molestias desde hace 11 meses, leves pero constantes, localizadas en el lado derecho de la cara, sensible al frío y al calor, y que a veces no la dejaba dormir. El dolor ha tenido aspecto variable por lo cual para descartar diversas patologías hubo de pasar por el otorrinlaringólogo, el médico internista, el psiquiatra, el neurólogo (el cual le puso tratamiento de las neuralgias sin resultado) y en dos ocasiones estuvo también en el dentista. Cuando acude a nuestra consulta, después de 11 meses, el dolor está más localizado en la hemiarcada inferior derecha. En la inspección y en la exploración radiográfica no se apreciaba nada especial (Fig. 5). Se recomendó un antibiótico (amoxilicina $500 \mathrm{mg} / \mathrm{ac}$. clavulanico $125 \mathrm{mg}$ ) y un antiinflamatorio (ibuprofeno $600 \mathrm{mg}$ ), pero la mejoría fue sólo transitoria. Cinco meses más tarde acude a consulta de nuevo con fractura mesio-distal del $2^{\circ}$ molar inferior derecho. La extracción de dicho diente dió 
por acabado con el dolor que había tenido durante 16 meses.

\section{DISCUSIÓN}

I dolor facial atípico, las neuralgias atípicas y otro tipo de odontalgias no siempre clasificables son en muchas ocasiones muy difíciles de determinar su origen. Algunos tipos de neuralgias idiopáticas ya conocemos que pueden estar determinadas por osteomielitis maxilares crónicas (1). Creemos al igual que otros autores (3), que el factor psicológico no es la causa prinicpal de este tipo de dolores y abría que buscar su etiología en alguna lesión de tipo orgánico. En los casos clínicos descritos mostramos como causa de este tipo de doloresx las fisuras de la corona del diente. Es algo difícil de diagnosticar mediante radiografía, pues suelen tener dichas fisuras una orientación sagital o parasagital al diente y no da lugar a la separación de los framentos hasta un tiempo muy posterior a esa primera consulta. Esto es lo que hace precisamente que el paciente busque solución en diferentes especialistas (4) sin que le determinen la causa de su dolor.

Diversos trabajos tienen determinada la importancia del tipo de fisuras en la corona del diente y su profundidad en cuanto al riesgo de caries (6), pero diriamos aquí que también tienen riesgo en cuanto a su posible transformación en fracturas. Entre una fisura profunda extendida mesio-distalmente con afectación de dentina y la fractura propiamente dicha de un fragmento de corona puede pasar mucho tiempo, ocasionando un dolor difuso, a veces poco localizado y que se etiqueta erroneamente como odontalgia atípica.

Los casos aquí presentados nos permiten poner de manifiesto como una de las causas de las odontalgias atípicas, a las fisuras de la corona dentaria extendidas hasta la capa de dentina, como paso previo a una fisura de dicha corona. Aconsejamos, pues, una exploración minuciosa de este tipo de lesión dental, para no pasarla desapercibida como posible causa de cualquier odontalgia atípica y proponemos como tratamiento de las mismas la endodoncia y colocación de una corona metal-porcelana de protección.

\section{BIBLIOGRAFÍA}

1. Peñarrocha Diago $M$, Peñarrocha Diago $M$, Bagán Sebastian JV. Cirugía oral y osteomielitis maxilares crónicas en pacientes con neuralgias trigeminales idiopaticas. Seguimiento clínico de 1 año. Av. en Odontoestom. 1994; 10 (8): 58797.

2. Olmedo MV, Gálvez R, Prieto $M$, Cañedo $P$, Moreno E, Vallecillo M, Galindo P. Nueva orientación clínica en el dolor facial atípico. Rev. Act. Odontoestomat. Esp. 1995; 55 (5): 43-7.

3. Graff-Radford SB, Solberg WK. Is atypical odontalgia a psychological problem? Oral Surg. Oral Med. Oral Pathol. 1993; 75: 579-82.

4. Kaiser C, Riva B, Pérez-Molina C, Sancho R. Consideraciones diagnósticas y clínicas sobre el dolor facial crónico y las afectaciones sinusales. Av. Odontoestom. 1996; 12 (suppl. B): 249-53.

5. Russell Vickers E, Cousins MJ, Walter S, Chisholm K. Analysis of 50 patients with atypical odontalgia. A preliminary report on pharmacological procedures for diagnosis and treatment. Oral Surg. Oral Med. Oral Pathool. Oral Radiol. Endod. 1998; 85: 24-32.

6. Estrela Sanchis F, Almerich Silla JM. Morfología de las fisuras oclusales: estudio a microscopía electrónica de barrido. Av. Odontoestom. 1996; 12: 343-6.

7. Padrós-Fradera E, Padrós-Serrat E, PadrósSerrat JL, Serrat-Caballería AM, Creus-Martínez $M$, Monterrubio-Berga $M$. El por qué y el cómo del tratamiento invasivo de las fisuras y el de la caries inicial. R.C.O.E. 1997; 2 (1): 23-40.

\section{CORRESPONDENCIA}

Dr. Jesús M. González-González

C/ Avila, $4,1^{\circ} \mathrm{A}$

37004 Salamanca (España) 